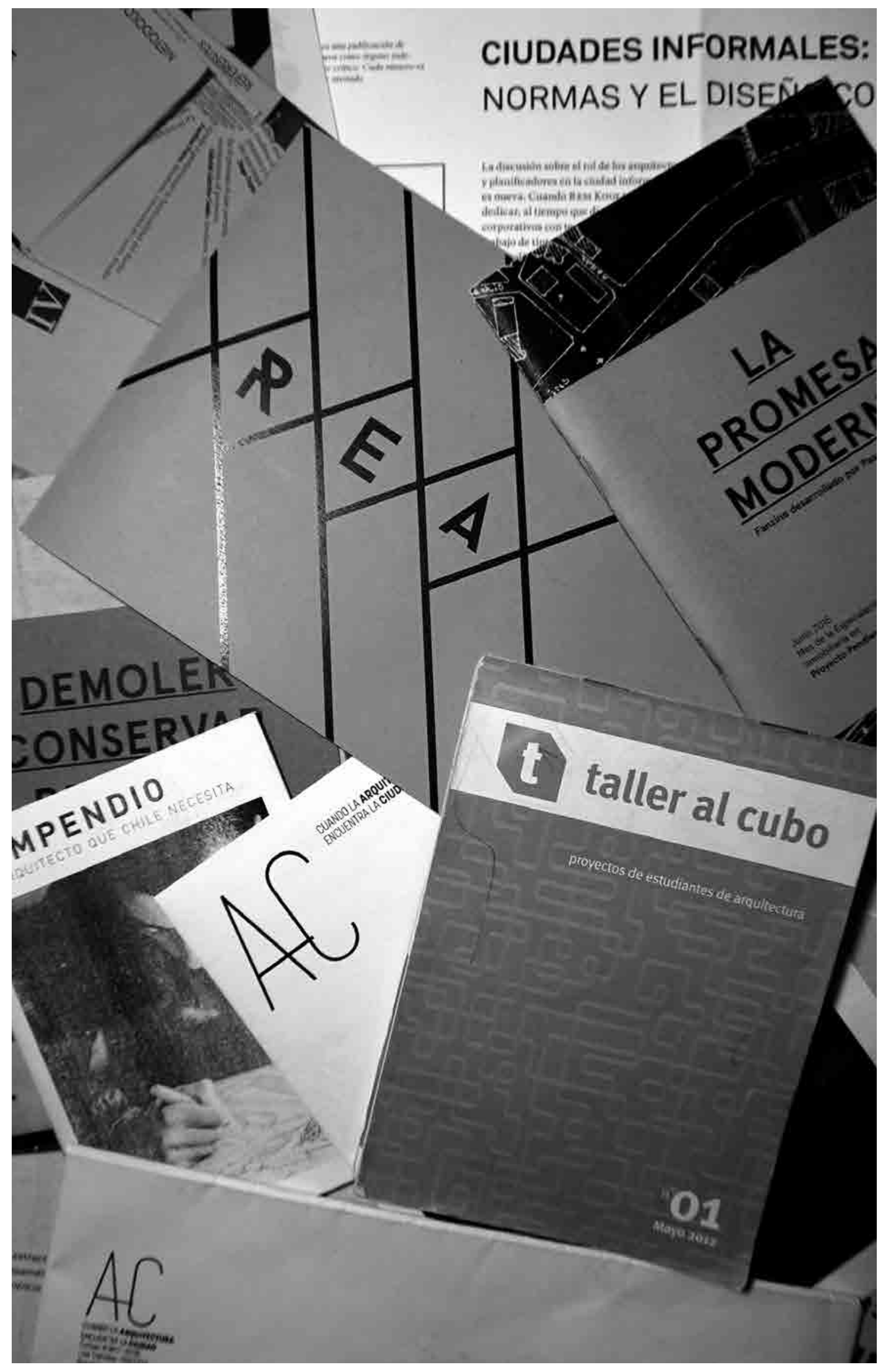

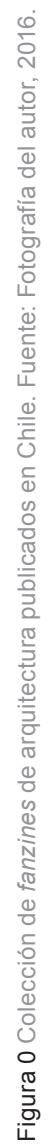



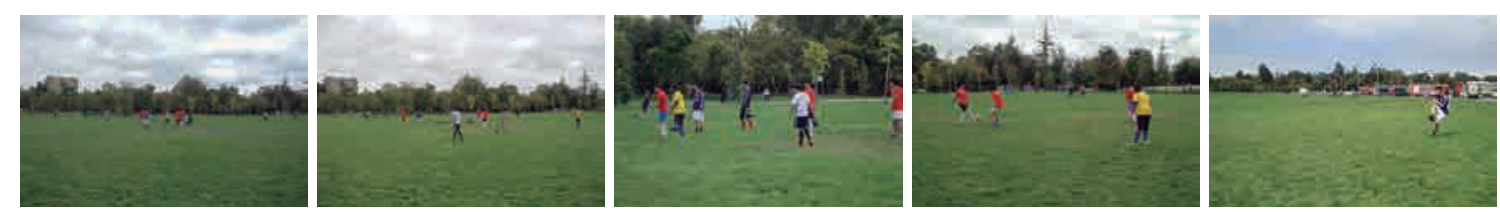

Fotos: Camila Muñoz

\title{
FANZINES DE ARQUITECTURA EN CHILE (2005-2016): EL ROL DE LAS REVISTAS INDEPENDIENTES DE ARQUITECTURA EN EL SIGLO XXI
}

\author{
ARCHITECTURE FANZINES IN CHILE (2005-2016): THE ROLE OF INDEPENDENT \\ ARCHITECTURE MAGAZINES IN THE 21ST CENTURY
}

\section{Christian Fierro Corral ${ }^{1}$}

RESUMEN

\begin{abstract}
Como disciplina compleja, en términos de sentido, forma y soporte, el estudio y análisis arquitectónico permite muchos medios para abordarse. La arquitectura existe gracias a la praxis como conexión fundamental; lo propio de la disciplina es el encuentro entre lenguaje arquitectónico y escrito. La disciplina se basa en hacer, pensar y declarar a través de diversas formas de representación, tales como las revistas de arquitectura, cuyo papel es transmitir tanto lo discursivo como lo proyectual mediante distintos formatos y soportes, definiéndose así como objetos con distintos niveles de información y como fuentes historiográficas en sí mismas. En ese ámbito, destacan los fanzines, publicaciones independientes de bajo costo que comunican inquietudes disciplinares en entornos limitados, donde la provocación y existencia en un pequeño marco histórico es lo necesario para transmitir ideas en discusiones abiertas, y donde la libertad en que se desarrollan permite discutir temáticas desligadas, generalmente, de las reglas entregadas por su contexto cultural e histórico. Este artículo presenta 10 casos chilenos del siglo XXI que el autor ha sistematizado y analizado, y ofrece conclusiones a nivel general sobre el rol de este tipo de publicaciones dentro de la disciplina, en base a las temáticas abordadas por ellas.
\end{abstract}

Palabras clave: arquitectura contemporánea, siglo XXI, revistas, historiografías, diseño editorial.

\section{ABSTRACT}

As a complex discipline, the study and analysis of architecture allows for many means to address meaning, form and support. Architecture exists thanks to praxis as a fundamental connection between ideas and practice; the discipline itself is the meeting of architectural and written language. Thus, architecture is based on doing, thinking and declaring through diverse forms of representation such as architecture magazines. Their role is to transmit elements of both discourse and projects by means of different formats and supports, thereby defining themselves as objects with different levels of information and as historiographical sources in and of themselves. In this area, fanzines stand out as independent, low-cost publications that communicate disciplinary concerns in a limited context, where provocation and existence in a small historical framework are necessary to transmit ideas in open discussions, and where the freedom with which they are created generally allows for the discussion of topics disconnected from the rules of their cultural and historical context. This article presents 10 Chilean cases from the 21st century that the author has systematized and analyzed, and offers general conclusions on the role of this type of publication within the discipline based on the topics they address.

Keywords: contemporary architecture, 21 st century, magazines, historiographies, editorial design.

Artículo recibido el 29 de agosto de 2016 y aceptado el 29 de noviembre de 2016 DOI: https://doi.org/10.22320/07196466.2016.34.050.07

[1] Revista de Arquitectura, Facultad de Arquitectura y Urbanismo, Universidad de Chile, Santiago, Chile.christian.fierro@ug.uchile.cl 
La arquitectura, como disciplina teórica y práctica, supone muchas maneras de ser estudiada. Por ser compleja en sentido, forma y soporte, su análisis se desarrolla entre lo teórico y lo práctico, con un sinfín de posibilidades. La arquitectura existe gracias a la praxis, aquel nexo fundamental entre idea y materialización, donde lo propio de la disciplina no es sólo el lenguaje arquitectónico ni el lenguaje escrito, sino su punto de encuentro. Así, ésta no se basa sólo en el hacer, sino también en el pensar y declarar, de ahí la importancia de cómo expresamos las ideas disciplinares mediante diversos medios de representación (Corvalán, 2014).

La producción editorial especializada en arquitectura es un trabajo que, recogiendo ambas dimensiones, manifiesta aquella condición teórico-práctica, cuya complejidad se traduce en puntos de vista divergentes, así como en formatos y soportes adaptados a cada caso. En este sentido, las revistas de arquitectura son esenciales en el sustento representacional que requiere la disciplina, tanto en el campo discursivo como operativo.

La revista, como producto editorial, tiene el propósito de ser evidentemente especializada, con una postura definida tanto por las opiniones manifestadas dentro de ella como por sus políticas editoriales. Asimismo, "el carácter de las revistas es plural y no individual como se da en los libros, contrastando y expresando la visión de diversos autores e instituciones" (Cruz y Durán, 2011:59). En particular, la revista de arquitectura es capaz de generar contenido propositivo y corresponde a un soporte de múltiples interpretaciones y formas de representación por su carácter teórico-práctico y diversidad temática. No otorga información sólo a través de textos; por ser compleja en términos de expresión busca también estímulos visuales a través de distintas maneras gráficas que permitan dar a entender sus planteamientos. Efectivamente, "las revistas de arquitectura corresponden a un tipo concreto de soporte que es la publicación periódica especializada [que destaca] como una fuente de carácter mixto en la que varios componentes, además del texto, hacen de ella un objeto complejo en forma y contenido, que permite diferentes niveles de información" (Hurtado, 2001:12).

Sobre las formas de actuar de las revistas de arquitectura, en cuanto al contenido generado, existe una gran diferencia con respecto a lo que se puede difundir mediante libros, como señalan Gutiérrez y Méndez: "[...] ningún libro, por abarcante que sea su enfoque, será capaz de suplir el caudal de información que nos brindan las revistas de arquitectura. Ello es así pues la revista tiene la oportunidad de ofrecer no solamente las evidencias de las obras realizadas, sino también todo el mundo de los proyectos que no lograron concretarse pero que en ellos quedaron reflejados" (2001: $6)$.

La revista de arquitectura se presenta como una fuente historiográfica en sí misma, permite niveles de entendimiento del pasado, otorga el espacio para la crítica del estado actual de la arquitectura y puede proyectar directrices o discutir ideas sobre el futuro de la disciplina: 
"[...] son un registro histórico de los temas que aborda, recogen las pulsaciones del momento, son testimonio espontáneo de ideas $y$, cuando corresponde, también lo son de imágenes. Tienen el mérito de la actualidad respecto de la época en que se publicaron. Dejan constancia del estado de la arquitectura. Las revistas definen un campo de estudio acotado, son el objeto de las cosas que se tratan. En ese sentido son fuente historiográfica". (Aguirre, 2012:21)

Las primeras revistas de arquitectura aparecen a fines del siglo XVIII en Europa Occidental, en países como Francia y Alemania, asumiendo la diversificación del periodismo especializado en arte y humanidades (Hurtado, 2001), a partir de las cuales otros países crearon medios de difusión sobre su propia creación arquitectónica durante el siglo XIX. Desde entonces, son fuente de todo lo que la disciplina genera o desecha, se toma del presente inmediato, del pensamiento crítico del pasado y genera ideas para el futuro, revelando proyectos que jamás se concretarán y exhibiendo obras en desarrollo.

Durante los siglos XX y XXI han existido diversos formatos y soportes para la difusión de ideas, proyectos y obras, "el valor que vemos al estudiar las revistas de arquitectura se basa en la contemporaneidad que representan, a que son de carácter periódico y sus temas se desarrollan a partir de los problemas propios de la época" (Cruz y Durán, 2011:58), operando a través de numerosos medios representacionales. En definitiva, se concibe aquí a las revistas de arquitectura como un producto capaz de ser un soporte de distintos niveles de información, pero además de inmenso valor en la formación de arquitectos y en el desarrollo de líneas discursivas, operacionales y de representación.

Las revistas de arquitectura publicadas durante el siglo XXI en Chile pueden categorizarse por su entidad responsable, existiendo así revistas académicas, publicadas por una institución universitaria o de carácter científico; gremiales, que representan a una asociación profesional; culturales, publicadas por instituciones culturales, públicas o privadas, en que la arquitectura forma parte de la producción artística; o independientes, como publicaciones de otras organizaciones, donde destacan los denominados fanzines: producciones de bajo costo y, generalmente, de bajo tiraje.

El fanzine supone pocos medios para su producción, su formato se adapta buscando efectividad en la comunicación en forma y contenido. Es utilizado, generalmente, por organizaciones independientes que entregan información sobre sus trabajos realizados o para generar discusiones en torno a diversas temáticas. Por su bajo tiraje, tienen una circulación restricta que prácticamente se comunica de mano en mano.

En un programa de postgrado de la Universidad de Princeton, guiado por Beatriz Colomina, se investigó sobre las revistas independientes desarrolladas en el mundo durante las décadas de 1960 y 1970, llamándolas little magazines. Colomina (2012) las define como dueñas de pequeñas utopías que no se rigen por las reglas físicas, sociales o intelectuales de su contexto, sino que generan pequeños mundos para desenvolverse. La investigación produjo dos resultados: la publicación del libro "Clip, Stamp, Fold. The Radical architecture of Little magazines $196 x$ to 197x" (2010), que expone a las mencionadas publicaciones independientes, explicando su importancia para la disciplina en contextos determinados; y la exhibición de la investigación mediante información, material impreso, réplicas de los artículos y charlas denominadas Little Talks, en diversas exposiciones alrededor del mundo. Una de ellas se presentó en Chile entre los días 7 de junio y 5 de julio del año 2013, gracias al trabajo curatorial de Fernando Portal, Pablo Brugnoli y Andrea Lathrop (Consejo Nacional de la Cultura y las Artes, 2013), quienes además de exponer la muestra en Chile crearon una investigación, publicada en el catálogo Editar para transformar. Publicaciones de arquitectura y diseño en Chile durante los años 60 y 70, en el marco de la exposición Clip/Stamp/ Fold, presentando los casos de las revistas AUCA, INTEC, $C A, C E D L A$ y la Cooperativa Amereida, mediante textos $y$ entrevistas, imágenes de la exhibición y fragmentos de las Little Talks (Brugnoli y Portal, 2015) (Figura 1).
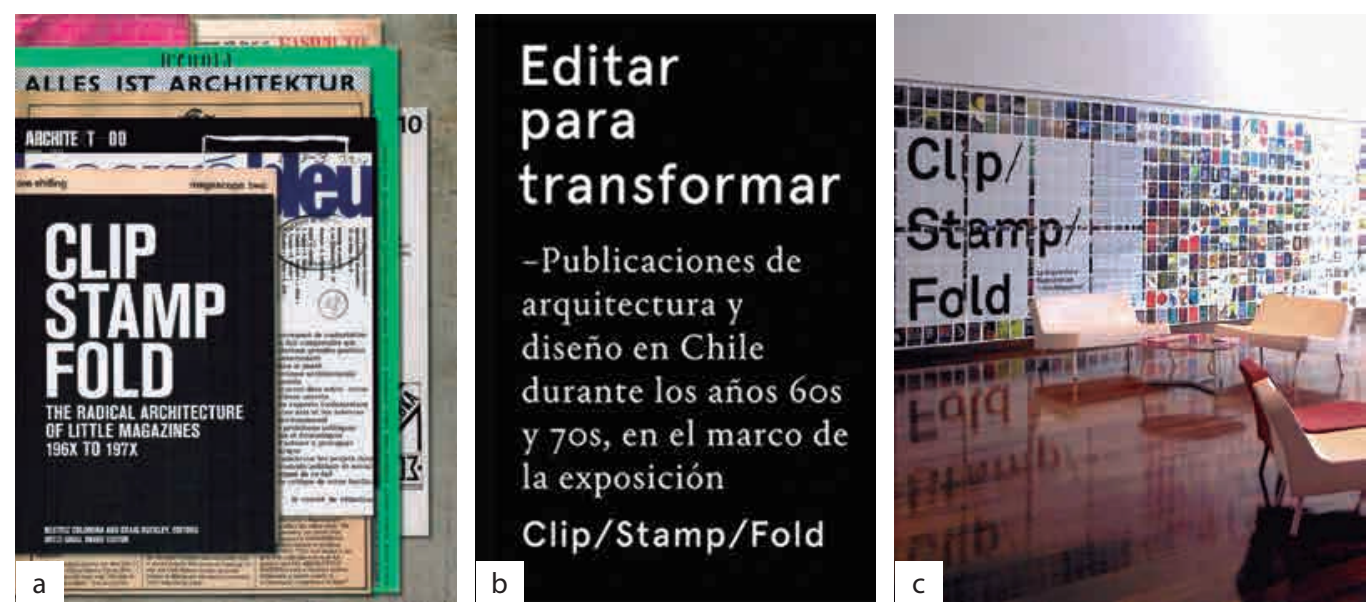

Figura 1 (a) Portada Clip, Stamp, Fold. The Radical architecture of Little magazines 196x to 197x. Fuente: Colomina y Buckley, 2010. (b) Portada catálogo Editar para transformar. Publicaciones de arquitectura y diseño en Chile durante los años 60 s y 70s, en el marco de la exposición Clip/Stamp/Fold. Fuente: Brugnoli y Portal, 2015. (c) Exposición "Clip/Stamp/ Fold" en el Centro Cultural Gabriela Mistral (Santiago). Fuente: http://metapoli.cl/blog/?p=4595, junio 2013. 
El estudio de los fanzines sigue vigente gracias al proyecto Archizines, que busca generar un archivo de publicaciones independientes creadas desde el año 2000, con un catálogo en línea y exhibiciones en diversas ciudades del mundo. Proyecto creado en la Architectural Association de Londres por Elias Redstone, que ha expuesto, en más de 30 ciudades, cerca de 80 revistas, principalmente europeas y estadounidenses $y$, en menor medida, latinoamericanas y asiáticas. Esta iniciativa fue también ofrecida en Santiago de Chile entre el 17 de octubre y 11 de noviembre del 2012, en BiblioGAM, con un montaje a cargo de Emilio Marín y Juan Carlos López, con exposiciones, charlas, un taller de fanzines y la presentación de la séptima publicación de la revista SPAM_arq; lo cual creó un ambiente diverso en contenidos, formatos y soportes (Gordon, 2012) (Figura 2).

\section{MÉTODO}

En el presente artículo se exhiben los 10 casos de fanzines publicados en Chile durante el siglo XXI, que se han podido sistematizar hasta el momento de la redacción de este texto. Sin embargo, se espera que ese corpus se incremente -pues constantemente están surgiendo nuevas publicaciones-, de modo que se constituya un archivo, que permita su consulta, en el entendido de que estos fanzines cumplen un objetivo autoimpuesto cuyo contexto, pertinencia e impacto varía según la agenda de publicación de cada caso, generando discusiones sobre múltiples temas propios de la arquitectura como de disciplinas afines. Así, el inicio de este período lo otorga la publicación de los primeros números de las revistas Cientodiez y SPAM_arq el año 2005, como los primeros casos registrados del siglo XXI, y el término corresponde al año 2016, en que se escribe el actual artículo. Se emplea una metodología que exhibe uno por uno los casos de estudio, presentando resultados específicos de manera cuantitativa y descriptiva, y concluyendo a nivel

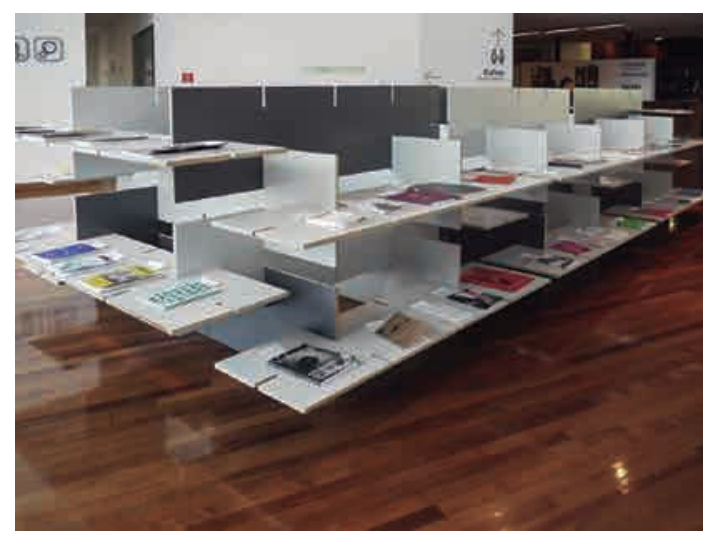

Figura 2 Exhibición Archizines en Chile. Fuente: Archizines, 2012

general, de manera cualitativa, sobre el rol de este tipo de publicaciones dentro de la disciplina, en base a las temáticas abordadas por ellas.

La motivación principal del estudio de estos casos es la construcción de un marco general en relación a ellos, que permita entender las diferencias y similitudes de sus proyectos editoriales y áreas temáticas tratadas en torno a la disciplina y sus múltiples campos de acción, con el fin de descubrir en sus discusiones y objetivos planteados la visión particular de cada editor o agrupación respecto a distintas problemáticas. Este trabajo constituye, por tanto, un análisis crítico de los fanzines publicados en Chile durante el siglo XXI, que los concibe como una fuente historiográfica sobre el desarrollo actual de distintas discusiones disciplinares. Su metodología se basa en un análisis mixto que presenta los casos de manera cuantitativa y cualitativa, a nivel particular y comparativo, con el fin de comprender sus formatos, las

CASO DE ESTUDIO

FANZINES PUBLICADOS

ESTADO ACTUAL

AÑOS EN VIGENCIA

\begin{tabular}{cccc}
\hline Cientodiez & 9 & Descontinuado & $2005-2008$ \\
\hline SPAM_arq & 7 & Vigente & $2005-$ \\
\hline Public Library & 3 & Descontinuado & $2008-2011$ \\
\hline Sin-título zine & 16 & Vigente & $2012-$ \\
\hline Taller al cubo & 1 & Descontinuado & 2012 \\
\hline La Paja Teórica & 6 & Vigente & $2013-$ \\
\hline Arquitectura Ahora & 11 & Vigente & 2015 \\
\hline $\begin{array}{c}\text { AC. Cuando la } \\
\text { arquitectura encuentra } \\
\text { la ciudad }\end{array}$ & 19 & Descontinuado & 2015 \\
\hline Mil m2 & 5 & Descontinuado & $2015-$
\end{tabular}




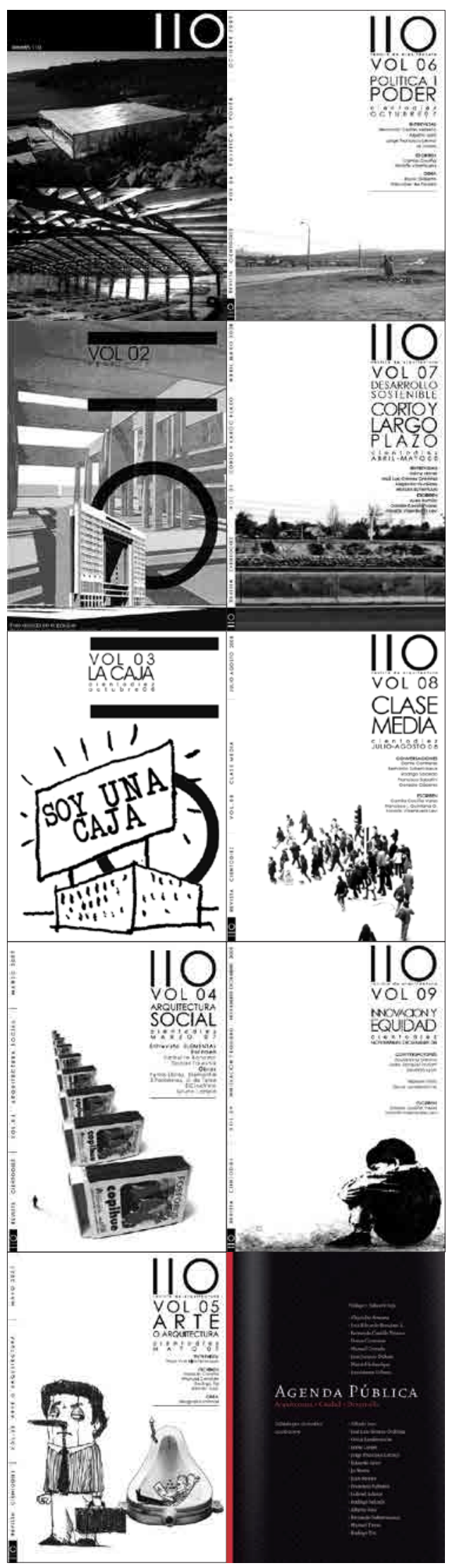

Figura 3 Portadas Cientodiez y portada Agenda Pública. Fuente: Cientodiez. organizaciones que los respaldan, los modos de gestión y discursos respectivos, permitiendo una apertura a nuevos casos de estudio que se encuentren latentes, bajo el carácter restricto mencionado anteriormente. Por ello, el corpus no se plantea como un catálogo estático sino que busca ser enriquecido con aquellas publicaciones que esperan ser encontradas (Tabla 1).

\section{CASOS DE ESTUDIO}

CIENTODIEZ. A cargo de Camila Cociña, Nicolás Valenzuela y Francisco Quintana, se publicó, entre 2005 y 2008, como una revista de discusión, difusión y crítica de la arquitectura desarrollada en Chile, mediante artículos, entrevistas y presentaciones de obras. Se distribuyó gratuitamente de manera impresa y digital, considerando un formato de 20 centímetros de alto por 110 centímetros de ancho. Llegó a editar 9 números, culminando con la publicación del libro Agenda pública, en mayo de 2009, que representó el cierre del trabajo realizado en la revista, el cual recopila todo el contenido generado, como fuente de consulta sobre distintas visiones en el campo profesional de la arquitectura y el estado de la crítica en los años que se desarrolló la revista (Figura 3).

SPAM. Editada por Pablo Brugnoli y diseñada por Kathryn Gillmore, desde el año 2005 explora las condiciones que entrega la ciudad mediante ensayos recibidos por convocatoria abierta, incluyendo miradas experimentales sobre el entorno urbano en base a una discusión que no represente una política institucional en estas materias. Forma parte del proyecto Archizines y su último número fue lanzado en el marco de la exposición que realizó dicho proyecto en Chile. La revista busca crear su propio espacio de discusión sobre temáticas que pueden o no estar relacionadas con la arquitectura, como escenario para imaginar y explorar diversos debates (Figura 4).

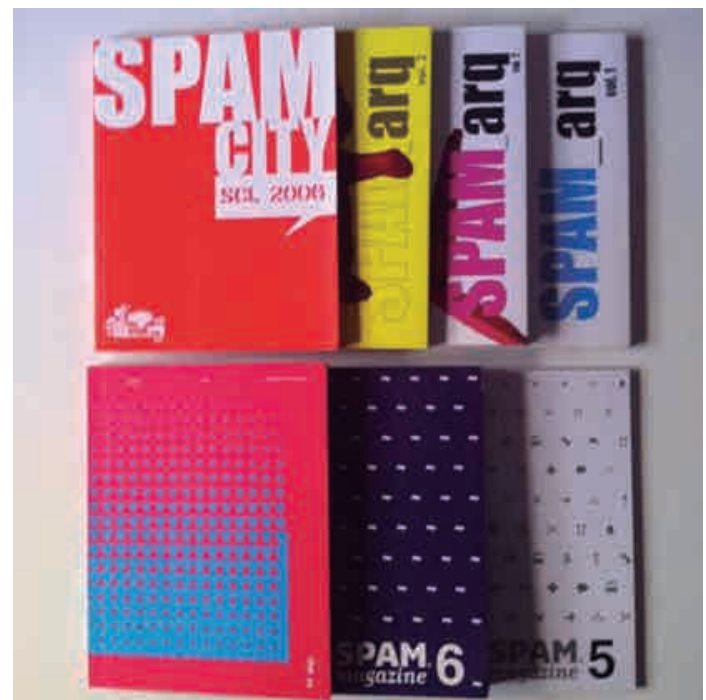

Figura 4 Revistas SPAM_arq, números 1 al 7. Fuente: SPAM_ arq, 2012 

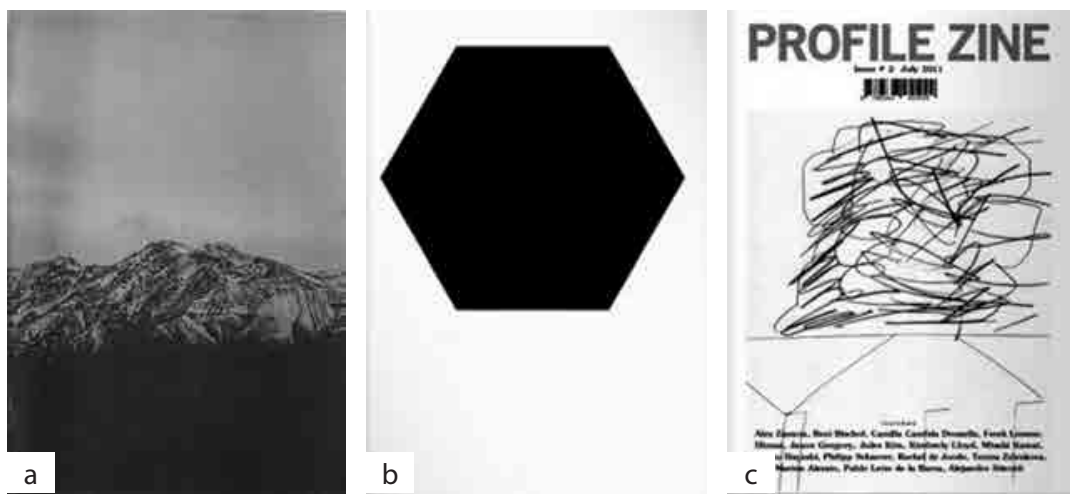

Figura 5 (a) Portada Casa de Todos. Fuente: Emilio Marín, 2008. (b) Portada Hexágono. Fuente: Emilio Marín, octubre 2008. (c) Portada Profile-zine, Fuente: Emilio Marín, julio 2011.

PUBLIC LIBRARY. Este proyecto, fundado en 2008 por el arquitecto Emilio Marín y el diseñador Diego Córdova como un ejercicio editorial independiente, se presenta como una exploración a través de distintos trabajos de arquitectos, diseñadores, artistas y fotógrafos y mediante múltiples acercamientos al quehacer disciplinar, publicando fanzines y posters. Fue incluido en Archizines, y en la exposición realizada en Chile, Public Library realizó un taller de fanzines siguiendo la lógica de la exploración de contenidos y formatos. Ha publicado Casa de todos (2008), en base a una casa en Santiago diseñada por la arquitecta Verónica Arcos; Hexágono (2008), en base al diseño del mobiliario urbano del Paseo Altamirano en Valparaíso hecho por Emilio Marín; y Profile-zine (2011) como una aproximación interdisciplinar con diversos actores (Figura 5).

SÍN-TíTULO ZINE. Incorporado en Archizines y editado en Iquique por Deseópolis, a cargo de Cristián Valenzuela Pinto, Sín-título Zine se plantea como una exploración narrativa y visual de lo que se encuentra marginado en el proceso de diseño arquitectónico y lo derivado de las prácticas arquitectónicas periféricas. En los primeros fanzines, The book of Towers (2012), The book of Mazes (2012) y The Book of Games (2012), se genera un intercambio narrativo-visual en torno a temáticas que escapan al ejercicio académico de la arquitectura, mientras en Mausoleum House (2012), Interrupted House (2013), House of Misunderstood (2013) y Uncanny House (2013) se explora desde el lenguaje escrito y visual propio del ejercicio proyectual en la disciplina. En Proyectos de Arquitectura se entrevista a arquitectos jóvenes, buscando una visión particular sobre el proceso de diseño arquitectónico, sólo mediante texto (Figura 6).

TALLER AL CUBO. Fundado y dirigido por Mario Ormazábal desde el año 2012, aparece como un espacio de exhibición de proyectos estudiantiles de pregrado y título, y como una vitrina abierta sobre concursos académicos y actividades de extensión dirigidas a estudiantes. Su último equipo editor estuvo compuesto por Nicolás Provoste, Héctor Antúnez, Pablo Espinoza, Enzo Vergara y Karen Troncoso. Su aparición fue mediante la publicación del fanzine Taller al Cubo como resultado del catálogo en línea de proyectos académicos, quedando luego el sitio web www.talleralcubo.com como único medio de difusión (Figura 7)

LA PAJA TEÓRICA. Iniciada en el año 2013, aparece como la publicación periódica del colectivo independiente Ciudad Atmosférica, como una respuesta crítica a la producción editorial académica. Si bien se presenta con un formato de bajo costo y mediante un soporte principalmente digital, la estructura de la revista se orienta más hacia la producción científica de la disciplina, con artículos publicados por convocatoria abierta, fundamentalmente sobre teoría y crítica. Su primer número apareció en mayo de 2014 y hasta la fecha lleva un total de 6 números (Figura 8).

ARQUITECTURA AHORA. Aunque su respaldo es la Universidad Diego Portales, la revista se presenta como un órgano independiente. Su director, desde el año 2014, es Mario Ormazábal y el editor, encargado de la temática y el contenido de cada número, es invitado y cambia número a número. Su formato es un póster tamaño $A 3$, con una estructura definida por la editorial -escrita por el editor invitado- que se acompaña de breves columnas de opinión, en el cual se emplea sólo el lenguaje escrito para representar la discusión temática llevada a cabo en cada número. Tanto por su formato como por las temáticas abordadas la revista posee un discurso que se modifica número a número, según un debate abierto desde la perspectiva de distintos autores. A partir de 2016 la revista se publica también en italiano, bajo el título de Architettura Adesso (Figura 9).

AC, CUANDO LA ARQUITECTURA ENCUENTRA LA CIUDAD. El ciclo de actividades dirigido por Pilar Pinchart, se inicia con la adjudicación de un Fondart el año 2015 $y$, con la arquitectura como excusa, busca dar lugar a distintos actores sociales y culturales en un espacio de intercambio multidisciplinar. En ese tenor, se realizaron 19 sesiones -entre marzo y diciembre de 2015-y encuentros entre arquitectos y otros profesionales en torno a discusiones que incluyeron directamente al público 


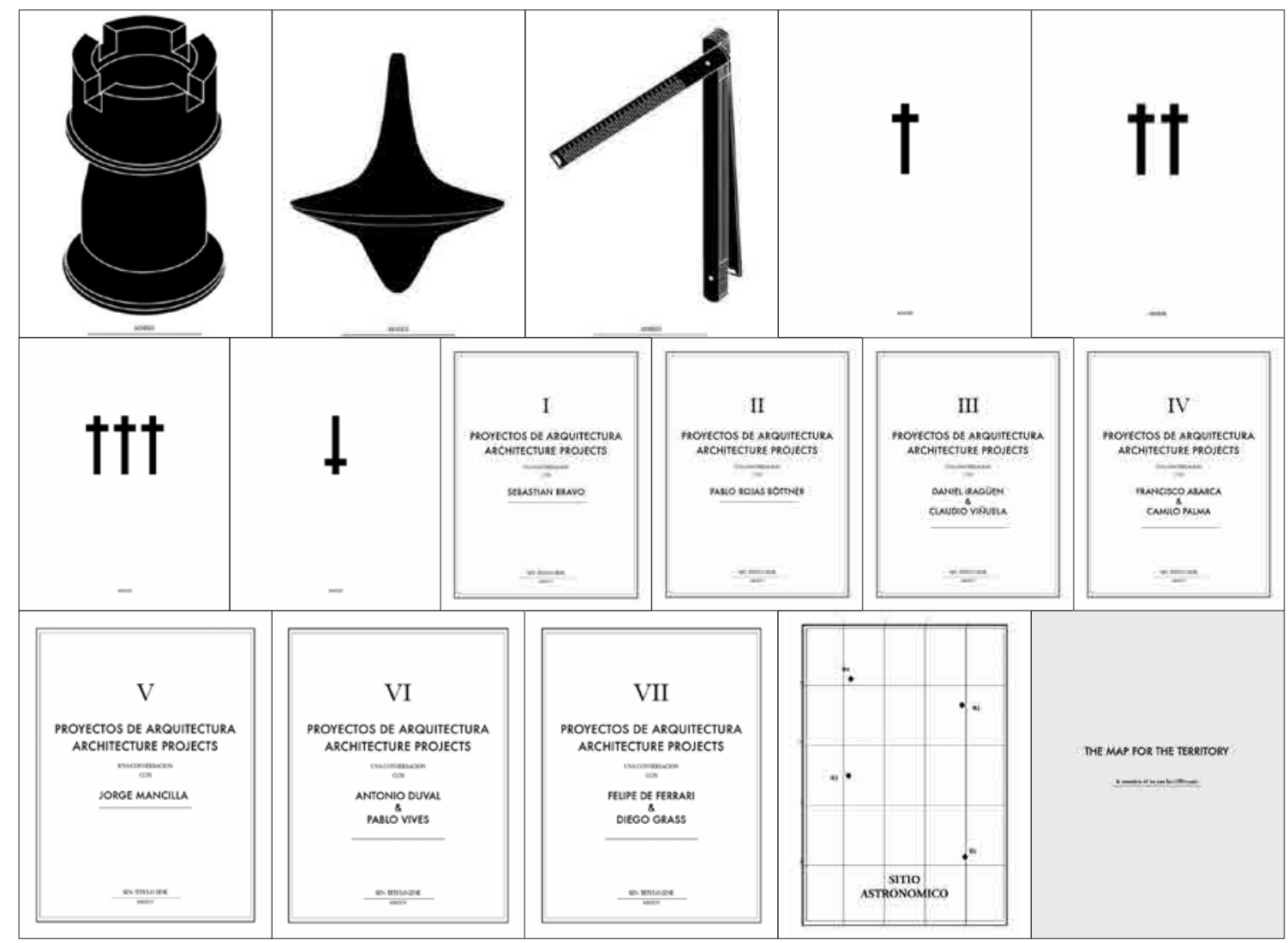

Figura 6 Portada de The book of Towers, The book of Mazes, The book of Games, Mausoleum House, Interrupted House, House of Misunderstood, Uncanny House, Proyectos de Arquitectura, Sitio Astronómico y The map for the territory. Fuente: Deseópolis.

\section{t taller al cubo}

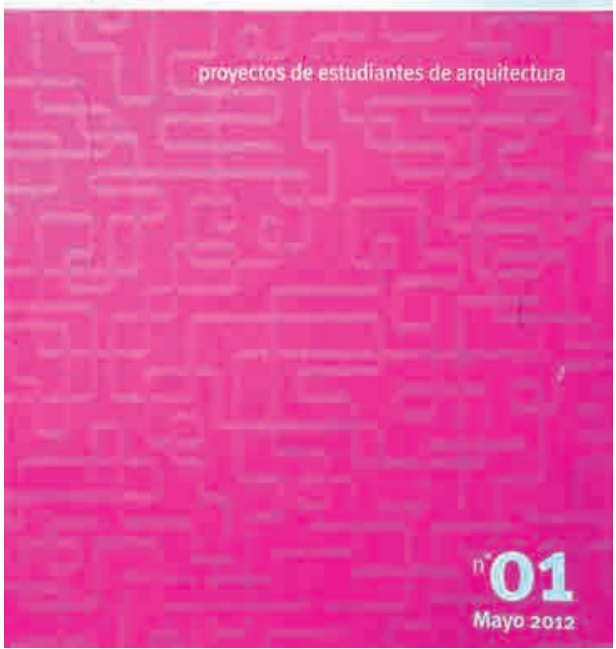

Figura 7 Portada Taller al Cubo número 1. Fuente: Taller al Cubo, mayo 2012

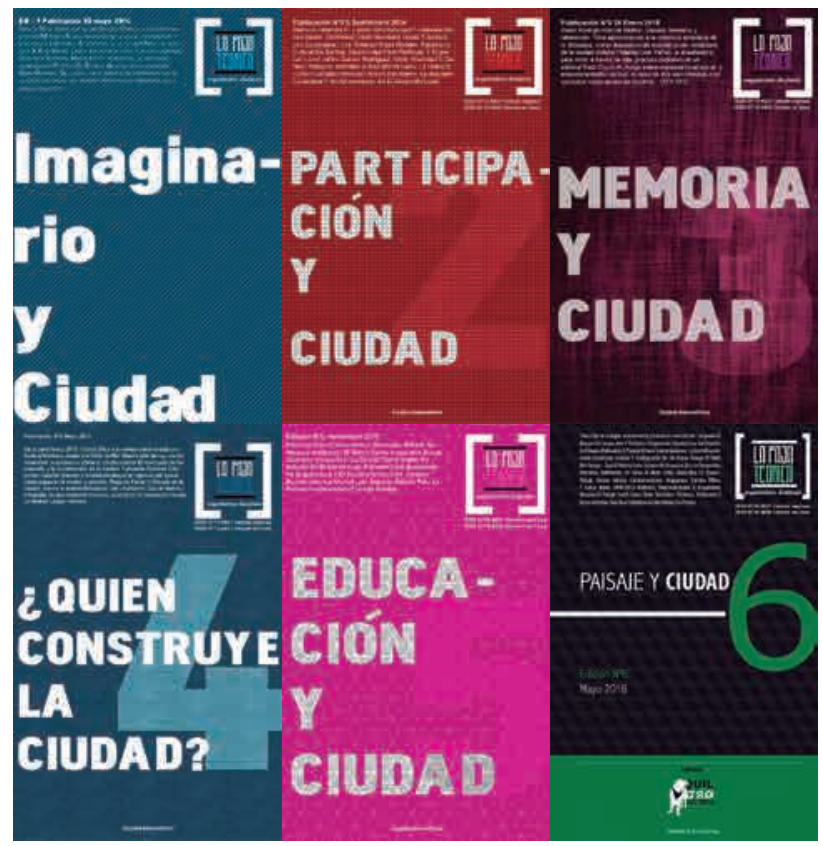

Figura 8 Portada La Paja Teórica número 1. Fuente: La Paja Teórica. 


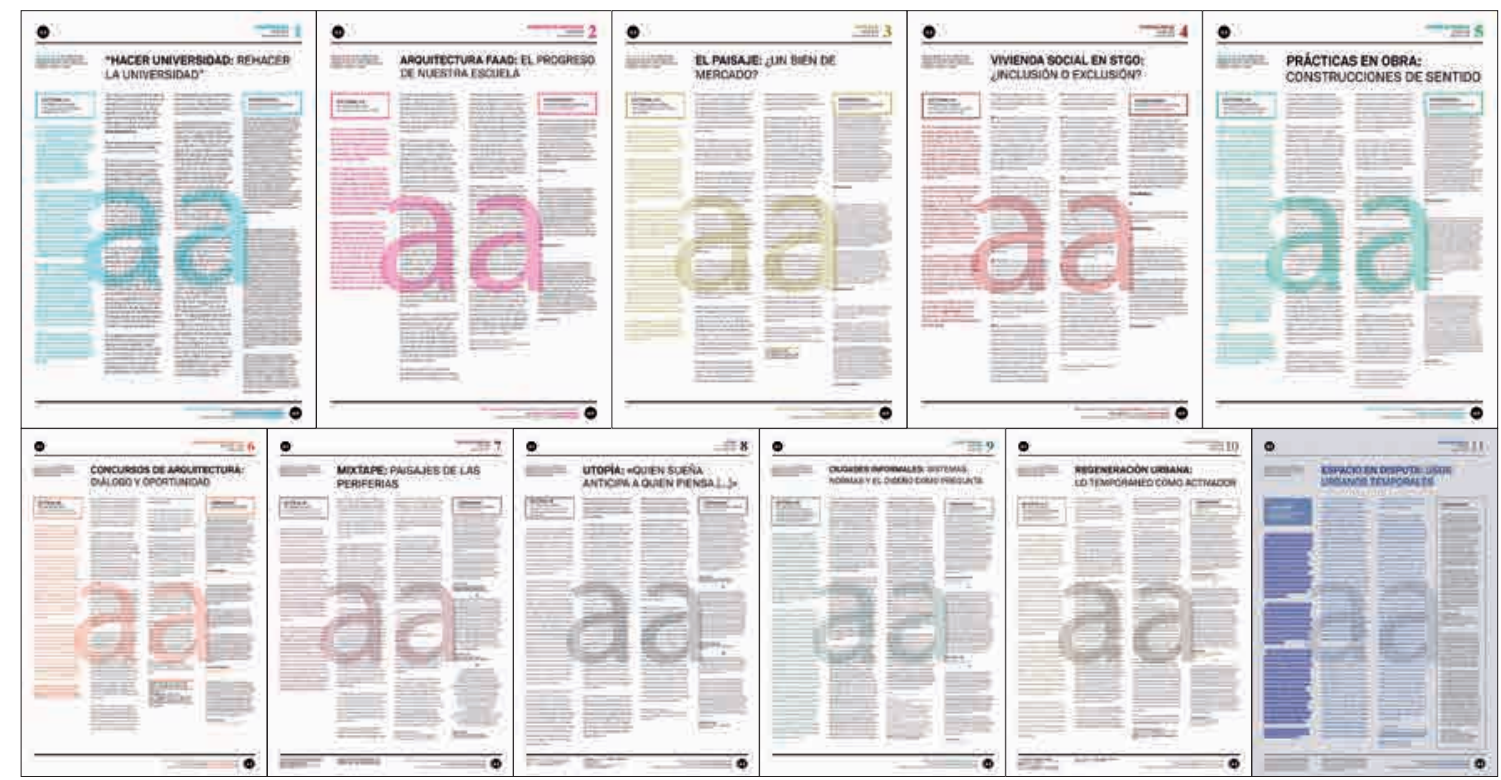

Figura 9 Portada Arquitectura Ahora. Fuente: Arquitectura Ahora.

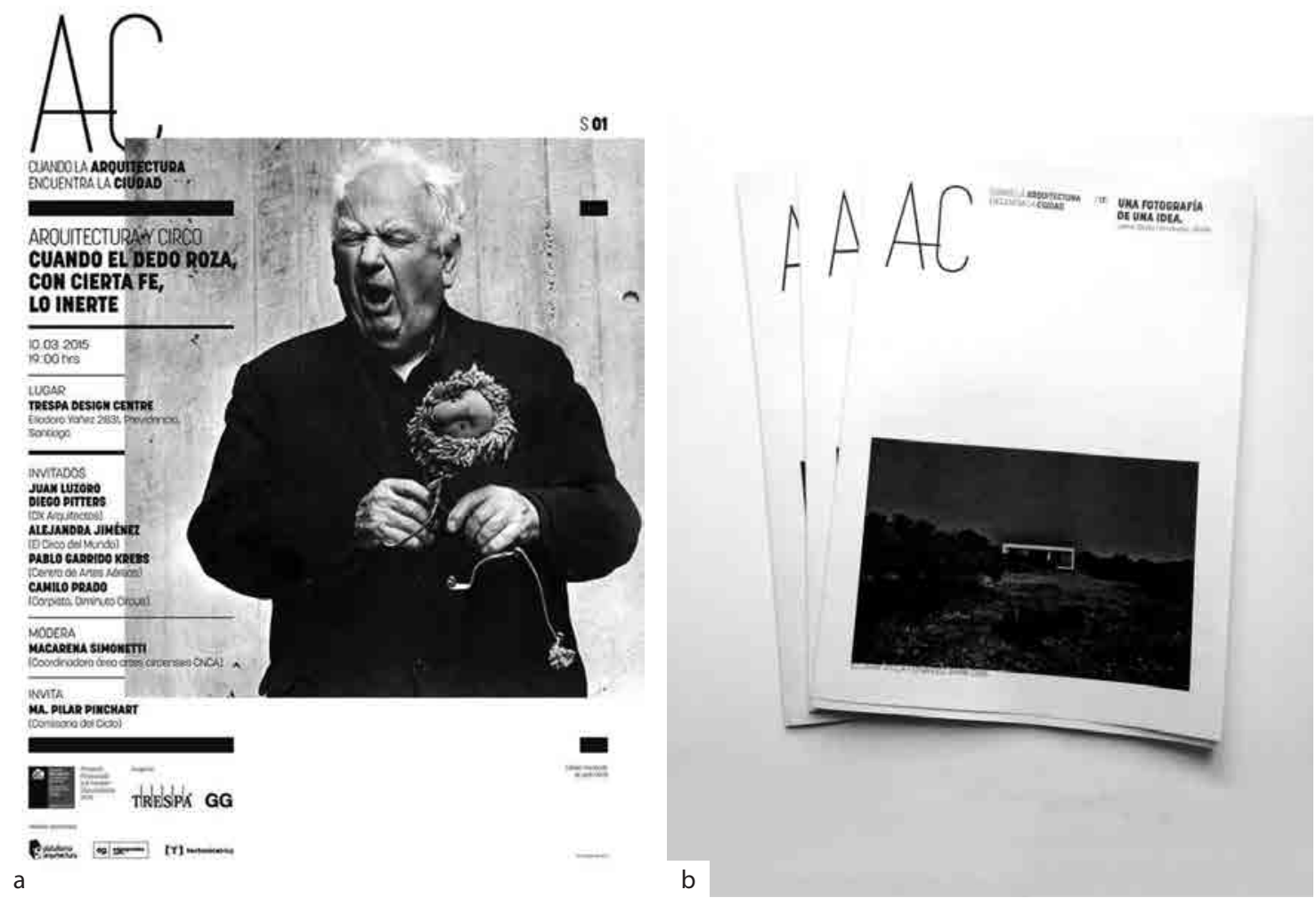

Figura 10 (a) Póster de la primera sesión de AC, Cuando la arquitectura encuentra la ciudad, 2015. (b) Fanzine Una fotografía de una idea, correspondiente a la sesión 17 de "Arquitectura y fotografía", octubre 2015 


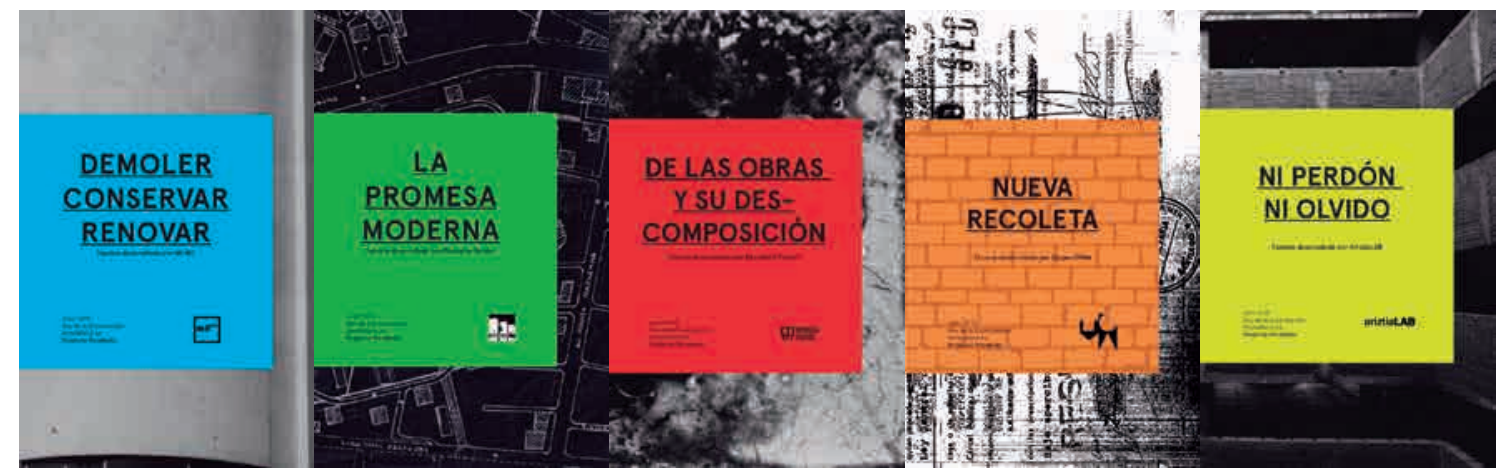

Figura 11 Portada Demoler conservar renovar, La promesa moderna, De las obras y su descomposición, Nueva Recoleta y Ni perdón ni olvido. Fuente: Mil m2.

asistente sobre distintas temáticas, especialmente desde la danza, el teatro, las artes visuales, el diseño, las ciencias, la literatura, la música, el cine y la fotografía. En cada sesión se hacía entrega gratuita al público de un fanzine con el resumen de la discusión expuesta por los profesionales invitados (Figura 10).

MIL M2. Mil m2 es una plataforma independiente de gestión, producción y creación cultural que nace en el año 2013 en busca de generar diversos proyectos para la apropiación colectiva de distintos espacios en búsqueda de nueva generación del conocimiento. En junio de 2015 presentaron el Mes de la Especulación Inmobiliaria, con un encuentro de distintas agrupaciones independientes de arquitectura, que buscan mediante la apropiación la transformación física y cultural de espacios en Santiago y Concepción. El resultado fue la publicación de 5 fanzines distintos, uno de cada agrupación: Mil m2, Pasarelas Verdes, República Portátil, Grupo Toma y Ariztía Lab; en todos ellos, el contenido es un debate permanente de diversas experiencias, entregando una visión sobre diversos problemas en que la apropiación y acción directa se presenta como solución (Figura 11).

RED DE ESTUDIANTES DE ARQUITECTURA. REA se presenta como una agrupación de creación colectiva, abierta a todos los estudiantes de arquitectura de Chile y Latinoamérica, que persigue generar espacios de discusión y crítica de la enseñanza. Su equipo es variable y se compone por estudiantes de distintas universidades, sean públicas o privadas. En abril de 2015, en el marco de la XIX Bienal de Arquitectura de Chile, publican el fanzine Compendio: el arquitecto que Chile necesita, compuesto de 13 textos que exponen la visión de estudiantes y arquitectos jóvenes precisamente acerca de la pregunta "¿cuál es el arquitecto que Chile necesita en el futuro?"

A partir de dicho texto, REA publica en noviembre de 2015 el número 0 de su Revista de Estudiantes de Arquitectura, Enseñanza de la arquitectura. Metodologías de taller, en la que se expone mediante entrevistas, opiniones y trabajos gráficos, la visión de estudiantes y académicos chilenos y latinoamericanos sobre la ense- ñanza dentro de los talleres de diseño arquitectónico. A raíz de esta publicación, y en el marco del lanzamiento de la revista ARQ número 91, REA lleva a cabo el conversatorio "Por qué / Cómo / Para qué publicar arquitectura", para debatir sobre el rol de las publicaciones de arquitectura y su función en la disciplina, que expone la visión de 7 invitados, representantes de distintas formas de publicar arquitectura.

REA se desempeñó también en el campo editorial al ser invitada a la II Bienal Iberoamericana de Arquitectura Académica (ARQA) en Cuenca, Ecuador, desarrollada entre el 6 y 10 de junio de 2016. Allí editó boletines, junto a la agrupación cuencana Maki, como una recopilación diaria de las actividades y la exposición de la muestra de la bienal, levantando información y publicándola en tiempo real; de ahí el título Taller Activo de Registro Proyectual, donde la información era generada por los estudiantes y académicos participantes (Figura 12). 

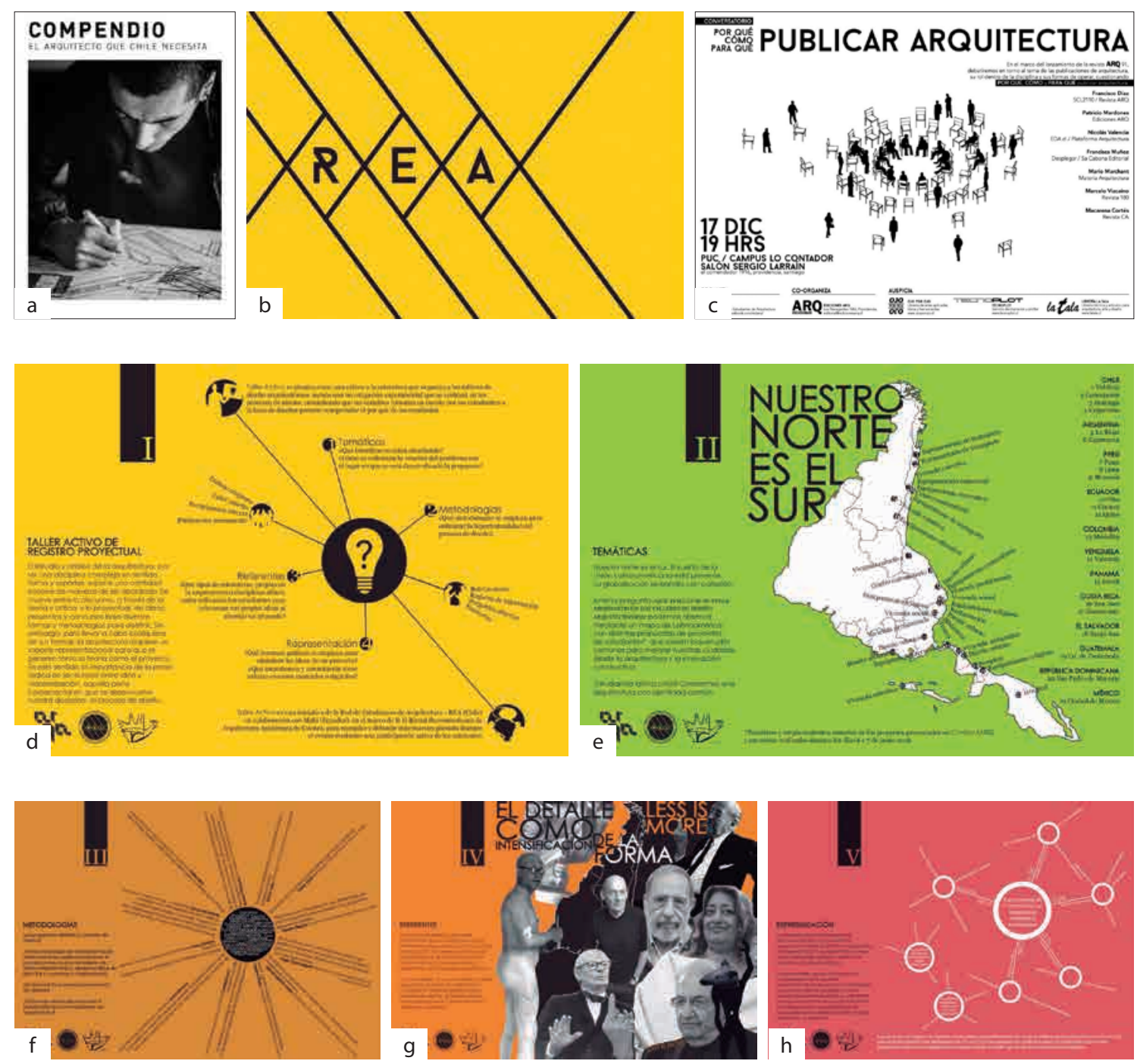

Figura 12 (a) Compendio: el arquitecto que Chile necesita. Fuente: www.estudiantesdearquitectura.cl, abril 2015. (b) Revista de Estudiantes de Arquitectura (REA) número O. Fuente: www.estudiantesdearquitectura.cl, noviembre 2015. (c) Afiche conversatorio "Por qué / Cómo / Para qué publicar arquitectura". Fuente: www.estudiantesdearquitectura.cl, diciembre 2015. (d) Boletín Taller Activo de Registro Proyectual número 1. Fuente: www.estudiantesdearquitectura.cl, junio 2016. (e) Boletín Taller Activo de Registro Proyectual número 2. Fuente: www.estudiantesdearquitectura.cl, junio 2016. (f) Boletín Taller Activo de Registro Proyectual número 3. Fuente: www.estudiantesdearquitectura.cl, junio 2016. (g) Boletín Taller Activo de Registro Proyectual número 4. Fuente: www.estudiantesdearquitectura.cl, junio 2016. (h) Boletín Taller Activo de Registro Proyectual número 5. Fuente: www. estudiantesdearquitectura.cl, junio 2016.

\section{RESULTADOS}

El panorama presentado en los 10 casos de estudio corresponde a la exhibición de 84 fanzines publicados en Chile entre los años 2005 y 2016. En ellos se puede entender tanto el carácter de diversidad temática como de formatos y soportes para ser publicados, así como su carácter de fuente historiográfica y reflejo de distintas discusiones disciplinares que se están desarrollando actualmente, tal como se planteaba en la introducción de este artículo. Las publicaciones fueron generadas por 14 agrupaciones de arquitectura, de las cuales 8 continúan trabajando de manera activa: Arquitectura Ahora, Ciudad Atmosférica, Mil m2, Pasarelas Verdes,
República Portátil, Grupo Toma, Ariztía LAB y Red de Estudiantes de Arquitectura (REA); todas las cuales realizan una labor de discusión y puesta en práctica de distintas inquietudes disciplinares (Tabla 2).

Los contenidos de cada fanzine entregan múltiples visiones de la arquitectura, en base a discusiones acerca de problemáticas contingentes o sobre pequeñas utopías y universos creados como escenario para debatir sobre temáticas amplias, en el sentido teórico o práctico que entrega la disciplina. Por ser independientes en su concepción, financiamiento, producción y difusión, se presentan también como pequeños panfletos de luchas reales o imaginarias sobre la apropiación urbana 


\begin{tabular}{|c|c|c|c|c|}
\hline CASO DE ESTUDIO & $\begin{array}{l}\text { FANZINES } \\
\text { PUBLICADOS }\end{array}$ & $\begin{array}{l}\text { FORMA DE } \\
\text { PUBLICACIÓN }\end{array}$ & $\begin{array}{l}\text { TEMÁTICAS } \\
\text { GENERALES }\end{array}$ & ÁREAS TEMÁTICAS \\
\hline \multirow[b]{2}{*}{ Cientodiez } & \multirow[b]{2}{*}{9} & \multirow{2}{*}{$\begin{array}{c}\text { Artículos, Entrevistas, } \\
\text { Obras }\end{array}$} & \multirow{2}{*}{$\begin{array}{l}\text { Crítica en torno } \\
\text { a la producción } \\
\text { arquitectónica } \\
\text { contemporánea }\end{array}$} & Crítica arquitectónica \\
\hline & & & & Urbanismo \\
\hline \multirow{4}{*}{ SPAM_arq } & \multirow{4}{*}{7} & \multirow{4}{*}{ Artículos, Obras } & \multirow{4}{*}{$\begin{array}{l}\text { Mirada crítica } \\
\text { y experimental } \\
\text { en torno a la } \\
\text { arquitectura } \\
\text { y la ciudad } \\
\text { contemporánea }\end{array}$} & Crítica arquitectónica \\
\hline & & & & Urbanismo \\
\hline & & & & Diseño \\
\hline & & & & Arte \\
\hline \multirow{3}{*}{ Public Library } & \multirow{3}{*}{3} & \multirow{3}{*}{ Obras, Ensayo Gráfico } & \multirow{3}{*}{$\begin{array}{c}\text { Presentación } \\
\text { experimental de } \\
\text { obras y proyectos de } \\
\text { arquitectura, artes y } \\
\text { diseño }\end{array}$} & Arquitectura \\
\hline & & & & Diseño \\
\hline & & & & Arte \\
\hline \multirow{3}{*}{ Sin-título zine } & \multirow{3}{*}{16} & \multirow{3}{*}{ Narrativa, Entrevistas } & \multirow{3}{*}{$\begin{array}{c}\text { Narrativa sobre } \\
\text { prácticas periféricas } \\
\text { derivadas del proceso } \\
\text { de diseño }\end{array}$} & Arquitectura \\
\hline & & & & Literatura \\
\hline & & & & Arte \\
\hline Taller al cubo & 1 & Proyectos & $\begin{array}{l}\text { Presentación } \\
\text { de proyectos } \\
\text { estudiantiles }\end{array}$ & Arquitectura \\
\hline \multirow{4}{*}{ La Paja Teórica } & \multirow{4}{*}{6} & \multirow{4}{*}{$\begin{array}{l}\text { Artículos, Obras, } \\
\text { Ensayo gráfico }\end{array}$} & \multirow{4}{*}{$\begin{array}{l}\text { Discusiones sobre } \\
\text { temáticas derivadas } \\
\text { del estudio de la } \\
\text { arquitectura y la } \\
\text { ciudad }\end{array}$} & Crítica arquitectónica \\
\hline & & & & Urbanismo \\
\hline & & & & Diseño \\
\hline & & & & Arte \\
\hline \multirow{2}{*}{ Arquitectura Ahora } & \multirow{2}{*}{11} & \multirow{2}{*}{ Opinión } & \multirow{2}{*}{$\begin{array}{c}\text { Crítica sobre } \\
\text { problemáticas } \\
\text { urbanas }\end{array}$} & Crítica arquitectónica \\
\hline & & & & Urbanismo \\
\hline \multirow{3}{*}{$\begin{array}{c}\text { AC. Cuando la } \\
\text { arquitectura } \\
\text { encuentra la ciudad }\end{array}$} & \multirow{3}{*}{19} & \multirow{3}{*}{$\begin{array}{l}\text { Opinión, Entrevistas, } \\
\text { Ensayo gráfico }\end{array}$} & \multirow{3}{*}{$\begin{array}{c}\text { Resúmenes de } \\
\text { jornadas de discusión } \\
\text { sobre la relación } \\
\text { de la arquietctura } \\
\text { con distintas artes y } \\
\text { humanidades }\end{array}$} & Arquitectura \\
\hline & & & & Diseño \\
\hline & & & & Arte \\
\hline \multirow{2}{*}{ Mil m2 } & \multirow{2}{*}{5} & \multirow{2}{*}{$\begin{array}{l}\text { Narrativa, Ensayo } \\
\text { gráfico }\end{array}$} & \multirow{2}{*}{$\begin{array}{c}\text { Trabajo de colectivos } \\
\text { de apropiación } \\
\text { colectiva de espacios } \\
\text { residuales }\end{array}$} & Arquitectura \\
\hline & & & & Urbanismo \\
\hline \multirow{3}{*}{$\begin{array}{l}\text { Red de Estudiantes } \\
\text { de Arquitectura (REA) }\end{array}$} & & & Enseñanza de la & Crítica arquitectónica \\
\hline & 7 & $\begin{array}{l}\text { Opinión, Entrevistas, } \\
\text { Ensayo gráfico }\end{array}$ & $\begin{array}{l}\text { arquitectura y sus } \\
\text { distintos campos de }\end{array}$ & Urbanismo \\
\hline & & & acción & Educación \\
\hline
\end{tabular}

Tabla 2 Temáticas abordadas en los casos de estudio. Fuente: Elaboración propia, 2016.

por parte de los editores o autores, invitando al debate al lector, como actor de estos nuevos espacios generados desde el mundo de las ideas y la discusión disciplinar. Los múltiples discursos teóricos y gráficos que plantean los casos de estudio, permiten comprender que efectivamente no se rigen por leyes de su contexto; al contrario, generan un pequeño mundo cuya libertad es autogenerada para desenvolverse, sin necesidad de responder a un rol específico, sino más bien en función de objetivos que varían caso a caso o, incluso, en cada publicación de un mismo caso de estudio, en función de su pequeña utopía y no necesariamente en respuesta al panorama arquitectónico en que están insertas.
Sintetizando, en los casos estudiados, se puede observar que 2 de ellos -Public Library y Sin-título zine- estudian las exploraciones formales en torno a la arquitectura, con 19 fanzines publicados (22,6\%), y solo uno -el único de Taller al Cubo-, se presenta como una vitrina de proyectos estudiantiles (1,2\%); mientras los 7 restantes -Cientodiez, SPAM, La Paja Teórica, Arquitectura Ahora, AC, Mil m2 y Red de Estudiantes de Arquitectura- buscan, a través de un total de 64 fanzines (76,2\%), en el debate, la discusión y el pensamiento crítico una manera de explorar distintas inquietudes en torno a la arquitectura, la ciudad, y su relación con distintas artes y humanidades. De esa manera, queda de manifiesto que estas últimas temáticas representan el área más abordada del período estudiado. 


\section{CONCLUSIONES}

La generación de un pequeño universo de ideas y discursos teóricos, gráficos y representacionales, permite que los fanzines se desarrollen en una libertad temática extendiendo inquietudes que no se encuentren forzosamente en boga en las publicaciones formales, y desligándose, por lo general, de las reglas entregadas por su contexto cultural e histórico. Asimismo, es precisamente esta libertad la que permite una búsqueda discursiva a pequeña escala, en contextos restrictos, sin necesidad de cumplir con un alto impacto, generando objetivos y agendas de publicación limitadas. En el caso específico chileno, durante el siglo XXI, el fenómeno estudiado se ha observado como un mecanismo de debate que permite la discusión en torno a temáticas propias de la crítica arquitectónica y urbanística, así como el cuestionamiento del rol de la disciplina ante inquietudes que se intersectan con otras áreas del conocimiento, como las artes y humanidades.

Así, los fanzines de arquitectura publicados en Chile, en lo que va del siglo XXI, se revelan como un juego de exploración que, según los medios disponibles, muestra y se adapta a distintos niveles de información, presentando un panorama del estado de la crítica arquitectónica, sea teórica o proyectual, generado desde agrupaciones independientes, en un espacio marginal de la producción disciplinar. De las 14 agrupaciones expuestas, 6 han desaparecido o se encuentran pausadas y 8 de ellas se encuentran activas, continuando la tarea de originar lugares de discusión mediante la apropiación y la práctica de acción directa, y a través de la utilización de nuevos medios digitales en favor de la difusión, la comunicación y el debate sobre arquitectura y ciudad, con todos los actores sociales implicados.

Estos proyectos editoriales tienen tal autonomía que su desaparición se da, en ciertas ocasiones, porque sus editores, coordinadores o autores, generalmente estudiantes $\mathrm{o}$ arquitectos jóvenes independientes $\mathrm{u}$ organizados en pequeñas agrupaciones, buscan otras alternativas para llevar a cabo proyectos de discusión teórica -como ocurrió con Cientodiez que cesó sus funciones para producir el libro Agenda Pública-, o proyectos de exploración más bien formal o práctica, como el caso de Public Library y el desarrollo de diversas propuestas realizadas por Emilio Marín. En otros casos, como Mil m2 o el ciclo de actividades $A C$, el objetivo fue generar números acotados, definiéndose desde un inicio la cantidad de publicaciones que se realizarían. Por su carácter independiente, en el proceso de generación de nuevas iniciativas editoriales no suele proponerse una larga duración ni un alto impacto, dejando la trascendencia de la publicación en manos de la libertad con que se desenvuelva dicho proyecto.

La utilización del fanzine como medio de comunicación de discusiones teórico-prácticas refleja su condición de productor colectivo de conocimiento, principalmente, bajo la lógica del debate permanente. El resultado es efímero, temporalmente distanciado de la trascendencia que se suele buscar en la disciplina, pero responde a esas pequeñas utopías que plantea Beatriz Colomina (2012), donde la provocación y la existencia dentro de un pequeño marco histórico es lo necesario para per- mitir el traspaso de ideas en discusiones abiertas. Por esto, los resultados muestran que más de un $75 \%$ de los fanzines publicados en el período esgrimen el debate, la discusión, la crítica arquitectónica o urbanística y el cuestionamiento al rol de la disciplina en la sociedad y su relación con otros campos del conocimiento, como temáticas centrales. Las pequeñas utopías tienen su lugar en el pensamiento y puesta en práctica de las agrupaciones independientes; de ellas depende, en suma, mantener viva la idea de que la arquitectura es una disciplina abierta, donde todas las discusiones poseen espacios para ser desarrolladas. 


\section{REFERENCIAS BIBLIOGRÁFICAS}

AGUIRRE, Max. La arquitectura moderna en Chile 19071942: revistas de arquitectura y estrategia gremial. $1^{\text {a }} \mathrm{ed}$. Santiago, Chile: Editorial Universitaria, 2012.

Archizines [en línea]. [Consultado 21 agosto 2016]. Disponible en: http://www.archizines.com/

Arquitectura Ahora [en línea]. [Consultado 21 agosto 2016]. Disponible en: https://issuu.com/arquitecturaahora/

BRUGNOLI, Pablo y PORTAL, Fernando (eds.). Editar para transformar. Publicaciones de arquitectura y diseño en Chile durante los años 60s y 70s, en el marco de la exposición Clip/Stamp/Fold. $1^{\text {a }}$ ed. Santiago, Chile: Capital Books, 2015.

Cientodiez [en línea]. [Consultado 21 agosto 2016]. Disponible en: http://wwwcientodiez.cl/

COLOMINA, Beatriz. Little magazines: small utopia. En García, l.; García-Diego, H. y Pozo, J. M. (coord.). Actas del Congreso Internacional: Las revistas de arquitectura (19001975) crónicas, manifiestos, propaganda. Pamplona, España: T6 Ediciones, 2012, pp.13-20.

COLOMINA, Beatriz y BUCKLEY, Craig. Clip Stamp Fold: the radical architecture of little magazines 196X to 197X. $1^{\mathrm{a}}$ ed. Barcelona, España: Actar, 2010.

CONSEJO NACIONAL DE LA CULTURA Y LAS ARTES (CNCA). Muestra "Clip/Stamp/Fold: La arquitectura radical de las little magazines 196x-197x" [en línea], 2013. [Consultado 21 agosto 2016]. Disponible en: http://www.cultura.gob.cl/agenda-cultural/muestra-clipstampfold-laarquitectura-radical-de-las-little-magazines-196x-197x/

CORVALÁN, Felipe. Representación arquitectónica y crítica proyectual. Nuevos campos de significación. Materia Arquitectura, 2014, n¹0, pp.26-35.

CRUZ, Sebastián y DURÁN, Francisco. Crítica de la crítica arquitectónica: análisis en revistas de arquitectura en Chile 2008-2011. Seminario de investigación inédito, Universidad de Chile, Chile, 2011.

Deseópolis [en línea]. [Consultado 21 agosto 2016]. Disponible en: http://deseopolis.tumblr.com/

Emilio Marín [en línea]. [Consultado 22 agosto 2016]. Disponible en: http://www.emiliomarin.cl/

GORDON, Katerina. Archizines llega a Chile. Plataforma Arquitectura [en línea]. 9 de octubre de 2012. [Consultado 21 agosto 2016]. Disponible en: http://www.plataformaarquitectura.cl/cl/02-196235/archizines-llega-a-chile

GUTIÉRREZ, Ramón y MÉNDEZ, Patricia. Revistas de arquitectura de América Latina: 1900-2000. 1a ed. San Juan, Puerto Rico: Cedodal, 2001.

HURTADO, Eva. Desde otra voluntad de permanencia. Las publicaciones periódicas de arquitectura. España 18971937 [en línea]. Tesis doctoral, Universidad Politécnica de Madrid, España, 2001. [Consultado 21 agosto 2016]. Disponible en: http://oa.upm.es/789/
La paja teórica [en línea]. [Consultado 22 agosto 2016]. Disponible en: https://issuu.com/lapajateorica/

Mil m2 [en línea]. [Consultado 21 agosto 2016]. Disponible en: http://www.milm2.cl/

Red de Estudiantes de Arquitectura, REA [en línea]. [Consultado 21 agosto 2016]. Disponible en: http://www. estudiantesdearquitectura.cl/

SPAM_arq [en línea]. [Consultado 21 agosto 2016]. Disponible en: http://www.spamarq.org/

Taller al cubo [en línea]. [Consultado 21 agosto 2016]. Disponible en: http://www.talleralcubo.com/ 